\title{
The endocrine-disrupting plasticizers will stay with us for a long time
}

\author{
Ji-Dong Gu* \\ School of Biological Sciences, The University of Hong Kong, Pokfulam Road, Hong Kong, China
}

\begin{abstract}
Many synthetic chemicals are widely used in our daily products and they are in constant contacts with humans through many different routes of exposure. In addition to the toxic environmental pollutants known with diminishing usage, plasticizers, a new class of emerging chemicals, are becoming of human health concerns increasingly. Microbial degradation of plasticizers is generally known, but the toxicity and other effects on human and animals are less well understood, especially in terms of reproductive development and endocrine-disrupting activity. Their major impact to Earth is the large quantities of them used on a daily basis and close contacts with biota of the biosphere. Knowledge of their impact shall be focused more specifically on developmental and endocrine system of animals than mortality in traditional toxicology as an end-point to better assess the threats to the biosphere. New directions on this research topic are presented to advance new knowledge in the future research and development.
\end{abstract}

Keywords: biodegradation; plasticizer; endocrine disruption; reproductive development; mineralization

* Correspondence to:

Ji-Dong Gu, School of Biological Sciences, The University of Hong Kong, Pokfulam Road, Hong Kong, China; E-mail: jdgu@ @ku.hk

Received: January 17, 2018; Accepted: February 7, 2018; Published Online: March 26, 2018

Citation: Gu, J.-D., 2018, Phytoremediation of cyanide and iron cyanide complexes and the mechanisms involved. Applied Environmental Biotechnology, 3(1), 61-64. http://doi.org/10.26789/AEB.2018.01.008.

Copyright: The endocrine-disrupting plasticizers will stay with us for a long time. (C) 2018 , Ji-Dong Gu. This is an Open Access article distributed under the terms of the Creative Commons Attribution-Noncommercial 4.0 International License, permitting all non-commercial use, distribution, and reproduction in any medium, provided the original work is properly cited and acknowledged.

Environmental organic pollutants in many different chemical forms through release and exposure can cause many environmental and human health problems (Schwarzenbach et al. 2006). Synthetic chemicals and polymeric materials are essential parts of our daily life now from high-strength construction materials to disposable packaging of various products to support the convenience of daily life. Human history on Earth has experienced from bronze, iron, and alloys to increasingly plastics and polymeric composite materials now to support the functioning of the society. Plasticizers together with filler, colorant and antimicrobial are indispensable chemical constituents of finishing commercial products, and they are required to allow the high molecular weight polymeric materials to be flexible for processing and molding (Gu, 2003; 2007; 2016b; 2017). To make any of the polymeric and plastic products, a suitable and selective plasticizer is always required so that the products can be made to the optimal designed requirements for the performance and durability to achieve (Gu et al., 1996; 2011; Kleerebezem et al., 1999; Kurane, 1997). Since plasticizers are not covalently bound to the polymeric resin, they release out of the products slowly into the ambient environment overt time $(\mathrm{Gu}$ and Wang , 2013). This property also allows results of microbial growth on surfaces of commercial plastics mistakenly taken as evidences of degradation of the plastic resin material incorrectly (Gu, 2016a, b; 2017).
Plasticizers are detectable in a wide range of environments (Gu and Wang, 2013; Kleerebezem et al., 1999) and human (Wenzel et al., 2018), which have serious environmental and human health concerns due to the wide occurrence of them and constant contact with human population (Chen et al., 2018; Kurane, 1997). They are endocrine active chemicals, which have been implicated for reproductive development and impairment through exposure in animals and humans (Corsini et al., 2018; Wenzel et al., 2018; Wong et al., 2017). Since these plasticizer chemicals are not comparatively speaking highly toxic to organisms in general judged by mortality as the criterion for evaluation, microorganisms in the environments including both bacteria and fungi can transform them relative easily to mineralization products (Fan et al., 2004; Li and Gu, 2006a, b; 2007; Li et al., 2005a, b; Luo et al., 2012). The contradiction between durability of the plastic products during service and subsequently quick degradation after disposable is not a simple one to be addressed scientifically or in applications. In addition, commercial plasticizers are widely used at enormous quantities with an increasing trend (Schwarzenbach et al. 2006) not only in plastics, but also in beverage for better suspension, and are detectable in human urine (Wenzel et al., 2018). With this in mind, a critical discussion on this topic of plasticizers is useful to illustrate the major shortcomings from identification of the scientific questions, experimental design, methods and analysis used 
to interpretation of the results obtained.

\section{Environmental Occurrence}

Since plasticizers coexist with plastics, and are detectable in environment and also biota as long as the plastics industries are in operation and manufacturing $(\mathrm{Gu}, 2017)$. All biota are exposed to them and the effects on the receiving organisms can be noticed. It should be pointed out that, strictly speaking, toxicity of them is not so high to individual organisms by mortality assessment, but effects of them on development and reproductive process can be much more pronounced (Chen et al., 2018; Wenzel et al., 2018; Wong et al., 2017). This is a unique characteristic of this class of environmental pollutants, different from the traditional toxic chemicals leading to mortality. In the degradation process, plasticizers can serve as a good source of carbon and energy source to ubiquitous microorganisms of the biosphere (Gu et al., 1996; 2011; Wang and Gu, 2006a, b; 2004; 2008), resulting in microbial growth, population, and enzyme activities observed, and also changes in functional groups of the chemical from microbial transformation. Commonly used plasticizers of the phthalate ester family are known to be degradable by a wide range of microorganisms, both aerobic and anaerobic (Cheung et al., 2007; Wang and Gu, 2006a; Gu and Wang , 2013; Xu et al., 2005, 2006). Degradation of them can be achieved with microorganisms in mixed cultures or purified culture from activated sludge, mangrove, wetland and non-polluted deepocean sediments (Gu and Wang , 2013; Li and Gu, 2006a; 2007; ?; ?; Xu et al., 2005, 2006). It should be mentioned here that the initial hydrolysis of ester bonds from phthalate diester to produce phthalate monoester and then phthalate in reaction sequence, but phthalate monoester is more toxic than the parent compound or the further degraded one, phthalate. Because of this, it is not only the parent compound phthalate diesters, but also phthalate monoesters, which are more toxic to biota than the corresponding parent one, shall be given adequate attention.

\section{Degradability of Plasticizers}

Plasticizers have a common structure of the aromatic moiety with dicarboxylic function groups onto the aromatic ring structure. Since both carboxylic groups can be esterified at the hydroxyl positions through substitution by alkane or aromatic side chain of common or different ones, there is a large collection of plasticizers for specific application in combination with the plastic resin and also application conditions (Kleerebezem et al., 1999; Kurane, 1997). In any scientific investigations on the degradation of organic chemicals, the chemistry of the plasticizer is both fundamental and very important, including solubility in water and physical stability under ambient conditions of investigation ( $\mathrm{Gu}, 2003$; 2007; 2016a, b; 2017). Many studies used overwhelmingly high concentrations of these chemicals in incubation investigations of aqueous or soils, the concentrations reported then are invalid and questionable. On the other hand, target chemical shall be the sole source of carbon and energy source in the cultural medium to link the degradation of the selective chemical with the growth of the microorganisms involved and implicated (Gu, 2016b). If exogenous sources of carbon or nutrients are added, the observed degradation is due to co-metabolism than specific transformation by the microorganisms involved. This approach does not allow further detailed in-depth investigation of the mechanisms and enzymes/genes involved. During the incubation in liquid medium, both decrease of the substrate chemical and the accumulation of biomass shall be shown simultaneously to allow establishment of biotransformation and biodegradation to be supported in the study $(\mathrm{Gu}, 2017)$. When dealing with soil or complex matrix, it is extremely important to assess the availability of the chemicals as effective concentration or activity from the conventional concentration by solvent extraction and chemical analysis to include the ageing effects in evaluating the fate of a chemical $(\mathrm{Gu}, 2016 \mathrm{a})$. Since soil is a highly heterogenous system, validation of biodegradation shall take into account of sorption and sequestration to better understand the contribution comprehensively by microorganisms and the extent of the degradation of a selective chemical in available form by biological processes involved from abiotic transformation or stabilization. Many researchers pay very little or no attention at all to these issues before the degradability of a chemical is claimed for utilization as the sole source of carbon and energy by microorganisms. Another largely neglected factor in soils or geological systems is the physical state of water and its availability, and biologically meaningful fraction of water is variable and different between different soils and geological materials. A better basic understanding of the lithosphere is crucial and vital to a scientific sound research to many investigators in this field. When chemical and degradation intermediates are quantified, mineralization cannot be warranted without significant biomass increase as a good indicator for substantial utilization of the substrate organic carbon to support the claim $(\mathrm{Gu}$, 2016b). A very necessary step for this is to conduct a mass balance calculation to account for the total $\mathrm{C}$ in the starting chemicals to the products, hopefully mineralized ones to draw a conclusive statement with strong scientific supports.

\section{Toxicity and Endocrine-disrupting Activity}

Environmental toxicity of chemicals is assessed for a wide range of environmental pollutants, especially pesticides/herbicides and persistent organic pollutants (Schwarzenbach et al. 2006). Plasticizers are not as lethal as these traditional toxic chemicals, and the toxicity of the former shall be evaluated accordingly by a different set of tests and criteria than the traditional mortality as an end point. This is critically important because different chemicals with variable effects shall be investigated to the relevance of their prominent effects than a fixed set of measurements, e.g., mortality as an 
example. For the plasticizers diesters of phthalates, the phthalate monoesters are more toxic than the corresponding parent phthalate diesters, but the monoesters are important degradation intermediates before the formation of phthalate prior to mineralization to mineralized products. Toxicity of phthalate monoesters is also higher than their parent diesters. Phthalate monoesters are all more soluble than their corresponding diesters in water, posing a much greater threat to biota. This issue on understanding of the chemistry of the chemical involved and then choice of the appropriate methods has not been addressed adequately and more attention shall be paid to similar issues to advance the research in this area and collect meaningful results on this topic.

Biodegradation of phthalate esters is initiated generally with hydrolysis reaction to cleavage of the ester bonds to form the common phthalic acid as reported before (Gu and Wang , 2013; Kurane, 1997; Xu et al., 2005, 2006). The hydrolysis of the ester bonds may be achieved by a single species of bacteria or specific cooperation of two different genus or species of bacteria to complete the degradation process ( $\mathrm{Li}$ and Gu, 2006a, b; 2007; Li et al., 2005a, b). Degradation can take place under aerobic or anaerobic conditions with different microbial communities involved to mineralize the plasticizer. The biochemical transformation steps involved for any selective plasticizer to the mineralization products involve a series of intermediate biochemical reaction steps before the complete degradation of the chemical is achieved (Gu and Wang , 2013). Based on this information, degradation of them should be focused on mineralization to minimize any negative impacts from the degradation intermediates to the biota and environmental quality.

\section{Future Perspectives}

The first issue on this research topic is that the plasticizers under investigation must be the sole sources of carbon and energy for microorganisms under conditions of investigation. Because complex cultural medium contains some or all of the followings: glucose, yeast extract, and/or nutrient broth to support the growth of a wide array of microorganisms, the biomass is supported by the availability of these nutrients and then transformation or degradation of the chemicals observed. As a result, the fundamental requirement in practice is to use defined mineral medium to allow a conclusions on degradation by microorganisms using the candidate chemical as the sole source of carbon and energy (Gu, 2017; Gu and Wang, 2013; Yip and Gu, 2016). This requirement can be fulfilled by enrichment culturing process when complex samples are used as inoculum, e.g., soils and sludge. Secondly, the physical and chemical limitations of the chemical must be considered so that the concentration used in the test must be below the maximum solubility in aqueous solution of culture medium. When test is monitored for substrate depletion over incubation time with inoculation of microorganisms, biomass shall be also assessed at the same time to collectively draw a conclusion based on a comprehensive set of data (Gu, 2016b; 2017). Thirdly, efforts shall be made on isolation and identi- fication of the microorganisms if pure culture of them is not used. In addition, identification of the degradation intermediates, and also the genes and/or proteins involved shall be made to confirm the degradation on a different dimension in addition to simply chemical concentration alone.

Furthermore, an abiotic control is always a must in parallel to the biologically active treatments to confirm the degradation process by microorganisms to eliminate any biases from chemical transformation, e.g., hydrolysis ( $\mathrm{Gu} 2017$; $\mathrm{Gu}$ and Wang , 2013). Many available reports do not provide an additional essential control, which is killed or sterilized inoculum to account for any effects from denatured proteins of the inoculum cells on the chemical in culture medium during the entirety of incubation. With a full consideration of these above, the experimental set up can obtain stronger convincing evidences on degradability of chemical by a pure culture or a stable consortium of microorganisms under laboratory conditions.

In conclusion, degradability of plasticizers is experimentally easy in set up, but the confirmation on degradation of them is not so easy to achieve for convincingly presentation of the results by microorganisms. Based on current available scientific information, degradation tests must follow the necessary guidelines and requirements so that the data generated are sound and valid without any doubts. With a full knowledge of the current available methodology and pitfalls, future research in planning on this topic can avoid these shortfalls to gain in-depth research advances through scientific investigations.

\section{Acknowledgements}

Research in my laboratory has been supported by grants from Hong Kong SAR Government and RGC-GRF.

\section{References}

Chen, C.H., Jiang, S.S., Chang, I.S., Wen, H.J., Sun, C.W. and Wang, S.L., 2018. Association between fetal exposure to phthalate endocrine disruptor and genome-wide DNA methylation at birth. Environmental Research, 162, 261-270. https://doi.org/10.1016/j.envres.2018.01.009

Cheung, J.K.H., Lam, R.K.W., Shi, M.Y. and Gu, J.D., 2007. Environmental fate of endocrine-disrupting dimethyl phthalate esters (DMPE) under sulfate-reducing condition. Science of the Total Environment, 381(1-3), 126-133. https://doi.org/10.1016/j.scitotenv.2007.03.030

Corsini, E., Ruffo, F. and Racchi, M., 2018. Steroid Hormones, Endocrine Disrupting Compounds and Immunotoxicology. Current Opinion in Toxicology, 10, 69-73. https://doi.org/10.1016/j.cotox.2018.01.006

Fan, Y., Wang, Y., Qian, P.Y. and Gu, J.D., 2004. Optimization of phthalic acid batch biodegradation and the use of modified Richards model for modelling degradation. International Biodeterioration \& Biodegradation, 53(1), 57-63. https://doi.org/10.1016/j.ibiod.2003.10.001

Gu, J.-D., 2003. Microbiological deterioration and degradation of synthetic polymeric materials: recent research advances. 
International Biodeterioration \& Biodegradation, 52(2), 6991. https://doi.org/10.1016/S0964-8305(02)00177-4

Gu, J.-D., 2007. Microbial colonization of polymeric materials for space applications and mechanisms of biodeterioration: a review. International Biodeterioration \& Biodegradation, 59(3), 170-179.

https://doi.org/10.1016/j.ibiod.2006.08.010

Gu, J.-D., 2016a. Biodegradation testing: so many tests but very little new innovation. Applied Environmental Biotechnology, 1(1), 92-95. http://dx.doi.org/10.18063/AEB.2016.01.007

Gu, J.-D., 2016b. More than simply bacterial growth curves. Applied Environmental Biotechnology 1(2),63-65. http://dx.doi.org/10.18063/AEB.2016.02.007

Gu, J.-D., 2017. Biodegradability of plastics: the pitfalls. Applied Environmental Biotechnology, 2(1), 59-61. http://dx.doi.org/10.26789/AEB.2017.01.008

Gu, J.-D. and Wang, Y. (2013) Microbial transformation of phthalate esters: diversity of hydrolytic esterases. pp. 313-345. In: Wong MH (ed), Environmental Contamination: Health Risks and Ecological Restoration. CRC Press, Boca Raton, Florida.

Gu, J.-D., Ford, T., Thorp, K. and Mitchell, R., 1996. Microbial growth on fiber reinforced composite materials. International Biodeterioration \& Biodegradation, 37(3-4), 197-204. https://doi.org/10.1016/S0964-8305(96)00035-2

Gu, J.-D., Ford, T., Thorp, K. and Mitchell, R., 2011. Chapter 30: Microbial degradation of polymeric materials. Pages 421-438. In: W Revie, (ed), The Uhlig Corrosion Handbook $\left(3^{\text {rd }}\right.$ ed), John Wiley \& Sons, New York.

Kleerebezem, R., Pol, L.W.H. and Lettinga, G., 1999. Anaerobic biodegradability of phthalic acid isomers and related compounds. Biodegradation, 10(1), 63-73. https://doi.org/10.1023/A:1008321015498

Kurane, R., 1997. Microbial degradation and treatment of polycyclic aromatic hydrocarbons and plasticizers. Annals of the New York Academy of Sciences, 829(1), 118-134. https://doi.org/10.1111/j.1749-6632.1997.tb48570.x

Li, J. and Gu, J.D., 2006a. Biodegradation of dimethyl terephthalate by Pasteurella multocida Sa follows an alternative biochemical pathway. Ecotoxicology, 15(4), 391-397. https://doi.org/10.1007/s10646-006-0070-8

Li, J. and Gu, J.D., 2006b. Biochemical cooperation between Klebsiella oxytoca Sc and Methylobacterium mesophilium $\mathrm{Sr}$ for complete degradation of dimethyl isophthalate. Water, Air, \& Soil Pollution: Focus, 6(5-6), 569-574. https://doi.org/10.1007/s11267-006-9041-6

Li, J. and Gu, J.D., 2007. Complete degradation of dimethyl isophthalate requires the biochemical cooperation between Klebsiella oxytoca $\mathrm{Sc}$ and Methylobacterium mesophilicum $\mathrm{Sr}$ isolated from wetland sediment. Science of the Total Environment, 380(1-3), 181-187.

https://doi.org/10.1016/j.scitotenv.2006.12.033

Li, J., Gu, J.D. and Pan, L., 2005a. Transformation of dimethyl phthalate, dimethyl isophthalate and dimethyl terephthalate by Rhodococcus rubber $\mathrm{Sa}$ and modeling the processes using the modified Gompertz model. International Biodeterioration \& Biodegradation, 55(3), 223-232.

https://doi.org/10.1016/j.ibiod.2004.12.003

Li, J., Gu, J.D. and Yao, J.H., 2005b. Degradation of dimethyl terephthalate by Pasteurella multocida Sa and Sphingomonas paucimobilis Sy isolated from mangrove sediment. International Biodeterioration \& Biodegradation, 56(3), 158-165. https://doi.org/10.1016/j.ibiod.2005.07.001

Luo, Z.H., Pang, K.L., Wu, Y.R., Gu, J.D., Chow, R.K. and Vrijmoed, L.L.P., 2012. Degradation of phthalate esters by Fusarium sp. DMT-5-3 and Trichosporon sp. DMI-5-1 isolated from mangrove sediments. pp.299-328 . In: Raghukumar C (ed), Biology of Marine Fungi. Springer-Verlag, Heidelberg, Germany. https://doi.org/10.1007/978-3-642-23342-5_15

Schwarzenbach, R.P., Escher, B.I., Fenner, K., Hofstetter, T.B., Johnson, C.A., Von Gunten, U. and Wehrli, B., 2006. The challenge of micropollutants in aquatic systems. Science, 313(5790), 1072-1077. https://doi.org/10.1126/science.1127291

Wang, Y. and Gu, J.D., 2006a. Degradation of dimethyl terephthalate by Variovorax paradoxus T4 and Sphingomonas paucimobilis DOS1 of the South China Sea. Ecotoxicology, 15, 549-557.

https://doi.org/10.1007/s10646-006-0093-1

Wang, Y.P. and Gu, J.D., 2006b. Degradation of dimethyl isophthalate by Viarovorax paradoxus strain $\mathrm{T} 4$ isolated from deep-ocean sediment of the South China Sea. Human and Ecological Risk Assessment, 12(2), 236-247. https://doi.org/10.1080/10807030500531521

Wang, Y.Y, Fan, Y. and Gu, J.D., 2004. Dimethyl phthalate ester degradation by two planktonic and immobilized bacterial consortia. International Biodeterioration \& Biodegradation, 53(2), 93-101. https://doi.org/10.1016/j.ibiod.2003.10.005

Wang, Y., Yin, B., Hong, Y., Yan, Y. and Gu, J.D., 2008. Degradation of dimethyl carboxylic phthalate ester by Burkholderia cepacia DA2 isolated from marine sediment of South China Sea. Ecotoxicology, 17(8), 845-852. https://doi.org/10.1007/s10646-008-0247-4

Wenzel, A.G., Brock, J.W., Cruze, L., Newman, R.B., Unal, E.R., Wolf, B.J., Somerville, S.E. and Kucklick, J.R., 2018. Prevalence and predictors of phthalate exposure in pregnant women in Charleston, SC. Chemosphere, 193, 394-402. https://doi.org/10.1016/j.chemosphere.2017.11.019

Wong, K.H. and Durrani, T.S., 2017. Exposures to Endocrine Disrupting Chemicals in Consumer ProductsA Guide for Pediatricians. Current Problems in Pediatric and Adolescent Health Care, 47(5), 107-118.

Xu, X.R., Li, H.B. and Gu, J.D., 2005. Biodegradation of an endocrine-disrupting chemical di-n-butyl phthalate ester by Pseudomonas fluorescens B-1. International Biodeterioration \& Biodegradation, 55(1), 9-15. https://doi.org/10.1016/j.ibiod.2004.05.005

Xu, X.R., Li, H.B. and Gu, J.D., 2006. Elucidation of n-butyl benzyl phthalate biodegradation using high-performance liquid chromatography and gas chromatographymass spectrometry. Analytical and Bioanalytical Chemistry, 386(2), 370-375. https://doi.org/10.1007/s00216-006-0627-7

Yip, K.C.W. and Gu, J.D., 2016. A novel bacterium involved in the degradation of 2-methylindole isolated from sediment of Inner Deep Bay of Hong Kong. Applied Environmental Biotechnology, 1(1), 52-63. http://dx.doi.org/10.18063/AEB.2016.01.008 